\title{
Severe weight loss during, but not before, simultaneous chemotherapy and radiation therapy for stage III and IV squamous cell carcinoma of the head and neck (SCCHN) is associated with increased survival
}

\begin{abstract}
Background: Severe weight loss commonly occurs before and during chemo-irradiation for Stage III and IV SCCHN. Nevertheless, the effects of severe weight loss on short and long term outcomes are unknown.

Objective: To evaluate effects of pre-treatment weight loss versus weight loss during chemotherapy/radiation for advanced operable SCCHN on toxicity, tumor response, recurrent tumor and survival

Methods: Records of 52 patients with Stage III and IV, clinically operable SCCHN who underwent primary high-dose radiotherapy and concomitant chemotherapy (cisplatin, $20 \mathrm{mg} / \mathrm{M}^{2} /$ four consecutive days during weeks 1,4 , and 7 of radiotherapy) (CTRT) were examined retrospectively in two groups: $<7.5 \%$ weight loss in three months during CTRT (NON-SEVERE; $\mathrm{n}=12$ ) and $>7.5 \%$ weight loss (SEVERE; $\mathrm{n}=41$ ). Data included tumor site, grade, stage, pre-CTRT weight loss, CTRT toxicity, response (CCR = Clinical Complete; $\mathrm{HCR}=$ Histologic Complete without residual tumor), surgeries, recurrence, and overall and disease-free survival. Statistical analysis: Chi-square, ANOVA, and Kaplan-Meier.

Results: Per study design, weight loss was greater in the SEVERE group versus NONSEVERE $(-17.3 \%+-7.9 \%$ versus $-2.9 \%+-4.2 \%, \mathrm{p}<0.0001)$. Pre-CTRT weight loss, age, stage, grade, toxicity of CTRT and post-CTRT surgery did not vary significantly. SEVERE tongue/hypopharynx primary site was increased $(66 \%$ versus $18 \%, \mathrm{p}<0.05)$. CCR and HCR were achieved in 5/11 (45\%) of NON-SEVERE and 29/41 (71\%) SEVERE. SCCHN recurred in 1/11 (9\%) NON-SEVERE and 12/41 (29\%) SEVERE patients. Kaplan-Meier Overall 60 month survival was $56 \%$ SEVERE and $14 \%$ NON-SEVERE $(p<0.001)$. DiseaseFree survival was $65 \%$ SEVERE and $22 \%$ NON-SEVERE $(p<0.001)$.

Conclusions: In spite of greater tongue/hypopharynx primaries and independent of pre-treatment weight loss, SEVERE Stage III/IV SCCHN CTRT toxicity, post-CTRT operations, and tumor recurrence did not increase. SEVERE CCR/HCR was excellent and SEVERE overall and disease-free survivals exceeded NON-SEVERE significantly. The pathophysiological mechanisms underlying these new and clinically important findings are not clear from the data.
\end{abstract}

Keywords: head and neck cancer, cisplatin, radiation therapy, squamous cell carcinoma, malnutrition, weight loss
Volume I3 Issue 3 - 202 I

Danielle Tamburrini, Casey Gene Sheck, Shawn Shaikh, Mckenzie Montana, Jessica Tyrrell, Michael A Davis DO, Jill Darminio, Gus J Slotman, and the Southern New Jersey Head and Neck Cancer Treatment Network Department of Surgery, Inspira Health Network, USA

Correspondence: Gus J Slotman, Department of Surgery, Inspira Health Network, 1505 West Sherman Avenue, Suite B, Vineland, NJ 08360, USA, Tel + I-856-64I-8635, Fax+I-856-64 I-8636, Email slotmang@ihn.org

Received: April 29, 202I | Published: June 14, 2021
Abbreviations: SCCHN, squamous cell carcinoma of the head and neck; CTRT, cancer treatment and research trust; CCR, clinical complete; HCR, histologic complete without residual tumor

\section{Introduction}

Severe weight loss commonly occurs before and during chemoradiotherapy for clinically operable Stage III and IV squamous cell carcinoma of the head and neck (SCCHN). The prevalence of malnutrition in patients with SCCHN is reported to be between 20$67 \% .{ }^{1}$ In a retrospective study of 1,562 patients, Pai et al. ${ }^{2}$, found that $>10 \%$ weight loss during treatment did not influence survival outcomes. ${ }^{2}$ Similarly, in a randomized trial of 224 patients, Ghadjar et al. ${ }^{3}$, reported that weight loss during treatment was not associated with survival. $^{3}$ In a pilot retrospective that did not include pre-treatment weight change, we correlated improved survival with higher weight loss. ${ }^{4}$ Conversely, pre-treatment weight loss of $>10 \%$ body weight correlated with decreased overall survival in multiple investigations. ${ }^{3,5}$

As discussed above, the interactions of pre-treatment malnutrition and of severe weight loss during concurrent chemotherapy and radiation therapy with short and long term outcomes in the treatment of SCCHN not clear from the data. The objective of this study, therefore, was to examine the interaction of pre-chemotherapy/radiation therapy nutritional status and weight loss during treatment of advanced, operable SCCHN on tumor response and overall survival. 


\section{Methods and materials}

With the approval of the Inspira Health Network IRB, medical records of 52 patients with advanced Stage III and IV SCCHN) for whom pre-treatment weight information was captured and who underwent the same high-dose radiotherapy and concomitant chemotherapy (cisplatin, 20mg/ $\mathrm{M}^{2}$ four consecutive days during weeks 1,4 , and 7 of radiotherapy) (CTRT) regimen as primary SCCHN treatment (6) were examined retrospectively. Pre-CTRT weight loss was recorded and intra-CTRT weight loss was calculated using pre and post CTRT weight.

CTRT chemotherapy consisted of preoperative cisplatin, $20 \mathrm{mg}$ / $\mathrm{M}^{2}$ for four consecutive days during weeks 1 , 4, and 7 of radiation therapy. Over the course of the study (2001-2011), the radiation therapy technique varied as the technology changed. In the earlier portion of the study, patients were treated with a regimen consisting of single daily fractionation with $6 \mathrm{MV}$ photons and 3D treatment planning followed by a boost, in which they were treated with a hyperfractionated (two fractions/day) regimen with concurrent chemotherapy. In 2006, patients were treated with normal fractionation to a higher total dose, between 70-74 Gy. In the latter part of the treatment study, the patients were treated with a field-within-a-field technique utilizing head and neck IMRT. Most treatment regimens were delivered with $6 \mathrm{MV}$ photons with either customized blocks or multi-leaf collimator generated blocks. Verification utilized port films, and later was changed to stereoscopic imaging followed by cone beam $\mathrm{CT}^{6}$

Patients were studied in two groups: Severe CTRT Weight-Loss (SEVERE), losing $>7.5 \%$ of pre-treatment weight in three months during CTRT; the Non-Severe CTRT Weight-Loss (NON-SEVERE), losing $<7.5 \%$ during CTRT. SEVERE and NON-SEVERE entry criteria followed NIH/Academy of Nutrition and Dietetics/ASPEN malnutrition and weight-loss definitions. ${ }^{1}$ Study variables included age at diagnosis, patient self-reported pre-CTRT weight loss, pre-CTRT weight, post-CTRT weight, body mass index (BMI), tumor site, grade, and stage, CTRT toxicity, clinical response, post-CTRT biopsy result, surgeries performed, recurrent cancer, overall survival (OS), and disease free survival (DFS). Clinical staging was by the classification of the American Joint Committee on Cancer Staging. ${ }^{7}$ Toxicity to treatment was determined according to the NCI Common Terminology Criteria for Adverse Events. ${ }^{8}$ Approximately 4-8 weeks after the last day of CTRT, patients underwent head and neck panendoscopy (direct laryngoscopy, esophagoscopy, and bronchoscopy) with biopsy of the primary tumor site. Clinical Complete Response (CCR) was defined as the resolution of visible tumor while Histologically Complete Response (HCR) was defined by negative biopsy results.

Statistical analysis used the chi-square equation, ANOVA, and Kaplan-Meier logarithmic rank test. ${ }^{9}$

\section{Results}

Pre-CTRT demographics are displayed in Table 1. Overall 44/52 patients (85\%) were men and 8/52 (15\%) were women, including $9 / 11$ (82\%) NON-SEVERE men and 35/41 (85\%) SEVERE men. Patientreported pre-treatment weight loss did not vary between NONSEVERE and SEVERE groups. Primary tumor site varied significantly between the two groups as tongue/hypopharynx comprised $66 \%$ of SEVERE patient and only $18 \%$ of NON-SEVERE (p 0.017). Cancer stage and age at diagnosis did not vary among SEVERE versus NONSEVERE.

Table I Pre-Treatment Demographics

\begin{tabular}{|c|c|c|c|}
\hline & Non-severe (s) & Severe (s) & P-Value \\
\hline Age at Diagnosis (yrs) & $61.1(10.2)$ & $59.6(10.3)$ & NS (I) \\
\hline Male & $59.8(9.4)$ & $58.3(9.8)$ & \\
\hline Female & $70.3(5.0)$ & $64.8(11.0)$ & \\
\hline Immediate Pre-CTRT Weight (Ibs) & I $47.5(33.1)$ & $185.2(42.3)$ & NS (0.82) \\
\hline Male & $153.8(34.0)$ & $189.3(42.5)$ & \\
\hline \multirow[t]{2}{*}{ Female } & |3| (23.8) & $168.3(37.3)$ & \\
\hline & $-4.8(6.2)$ & $-32.6(19.4)$ & $<.0001$ \\
\hline Weight Change During CTRT (Ibs) & $-4 . .6(6.7)$ & $-33.1(20.6)$ & \\
\hline Male & $-5.3(4.5)$ & $-30.5(12.8)$ & \\
\hline \multicolumn{4}{|l|}{ Female } \\
\hline Self-Reported Pre-Treatment Weight Loss & $-22.5(10.4)$ & $-18.1(16.8)$ & \\
\hline Male & $-24.3(11.4)$ & $-13.9(13.5)$ & NS (0.33) \\
\hline Female & $-17.0(0)$ & $-45.5(7.5)$ & \\
\hline Clinical Stage & NON-SEVERE (\%) & SEVERE (\%) & NS (0.42) \\
\hline III & 4/II (36\%) & $|3 / 4|$ (32\%) & \\
\hline IV & $0 / I I(0 \%)$ & $5 / 4 I(12 \%)$ & \\
\hline IVa & $6 / 11$ (55\%) & $|3 / 4|$ (32\%) & \\
\hline IVb & 0/II (0\%) & $4 / 4 \mid$ (9.7\%) & \\
\hline IVc & $0 / 1 \mathrm{I}(0 \%)$ & $|/ 4|(2 \%)$ & \\
\hline
\end{tabular}

Citation: Tamburrini D, Sheck CG, Shaikh S, et al. Severe weight loss during, but not before, simultaneous chemotherapy and radiation therapy for stage III and IV squamous cell carcinoma of the head and neck (SCCHN) is associated with increased survival.J Otolaryngol ENT Res. 2021;13(3):54-58. DOI: 10.15406/joentr.2021.13.00491 
Table Continued..

\begin{tabular}{llll}
\hline & Non-severe (s) & Severe (s) & P-Value \\
\hline Unknown & $\mathrm{I}$ & 5 & \\
Primary Location & & & 0.017 \\
Oral/Pharynx & & & \\
Tongue/Hypopharynx & $5 / 11(45 \%)$ & $7 / 41(17 \%)$ \\
Larynx & $2 / 11(18 \%)$ & $27 / 41(66 \%)$ & \\
& $4 / 11(36 \%)$ & $7 / 41(17 \%)$ \\
\hline
\end{tabular}

Results during and following CTRT are listed in Table 2. PostCTRT weight loss between males and females in the NON-SEVERE group versus the SEVERE group varied significantly $(\mathrm{p}<0.001)$
CTRT toxicity, surgeries performed, CCR, and HCR, and recurrent cancer did not vary significantly.

Table 2 Response to Pre-operative CTRT

\begin{tabular}{|c|c|c|c|}
\hline & Non-Severe (\%) & Severe (\%) & P-Value \\
\hline \multicolumn{4}{|l|}{ Toxicity to CTRT } \\
\hline $\mathrm{NCl}$ toxicity grade & & & NS $(0.27)$ \\
\hline I & 4/II (36\%) & $|9 / 4|(46 \%)$ & \\
\hline 2 & $3 / I I(27 \%)$ & |l/4| (27\%) & \\
\hline 3 & $3 / 11(27 \%)$ & $\mid \mathrm{I} / 4 \mathrm{I}(27 \%)$ & \\
\hline 4 & I/II (9\%) & $0 / 4 \mathrm{I}(0 \%)$ & \\
\hline \multicolumn{4}{|l|}{ Tumor response } \\
\hline \multicolumn{4}{|l|}{ Clinical Response } \\
\hline Clinically complete & $5 / 11(45 \%)$ & 29/4I (7I\%) & NS $(0.13)$ \\
\hline Partial & $5 / \mathrm{II}(45 \%)$ & $10 / 4 \mid(24 \%)$ & \\
\hline Unknown & I & 2 & \\
\hline \multicolumn{4}{|l|}{ Biopsy Result } \\
\hline Histologically complete & $5 / 11(45 \%)$ & 29/4I (7I\%) & NS $(0.13)$ \\
\hline Residual Disease & $5 / 11(45 \%)$ & $|0 / 4|(24 \%)$ & \\
\hline Unknown & I & 2 & \\
\hline \multicolumn{4}{|l|}{ Recurrence } \\
\hline No Reoccurrence & $10 / 11$ (91\%) & $26 / 4 \mid(63 \%)$ & NS $(0.14)$ \\
\hline Reoccurrence & I/II (9\%) & $|2 / 4|(29 \%)$ & \\
\hline -Distant & 0 & 2 & \\
\hline -Local & 1 & 10 & \\
\hline Unknown & 0 & 3 & \\
\hline
\end{tabular}

Overall survival and Disease-Free Survival are displayed in Figure 1. Kaplan-Meier 60-month overall survival was 56\% in the SEVERE group and only $14 \%$ in the NON-SEVERE group $(\mathrm{p}<0.001)$. Kaplan-
Meier 60-month disease-free survival was observed in $65 \%$ of the SEVERE group and only $22 \%$ of the NON-SEVERE group (p $<0.001)$. 

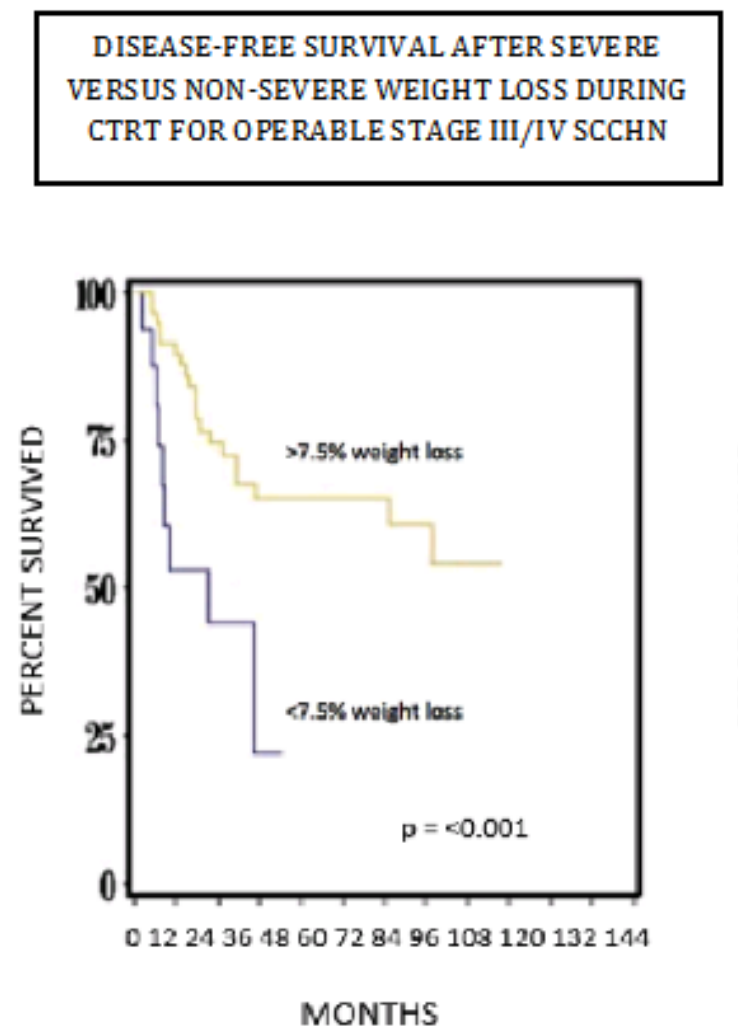

\section{OVERALL SURVIVAL AFTER SEVERE VERSUS NON-SEVERE WEIGHT LOSS DURING CTRT FOR OPERABLE STAGE III/IV SCCHN}

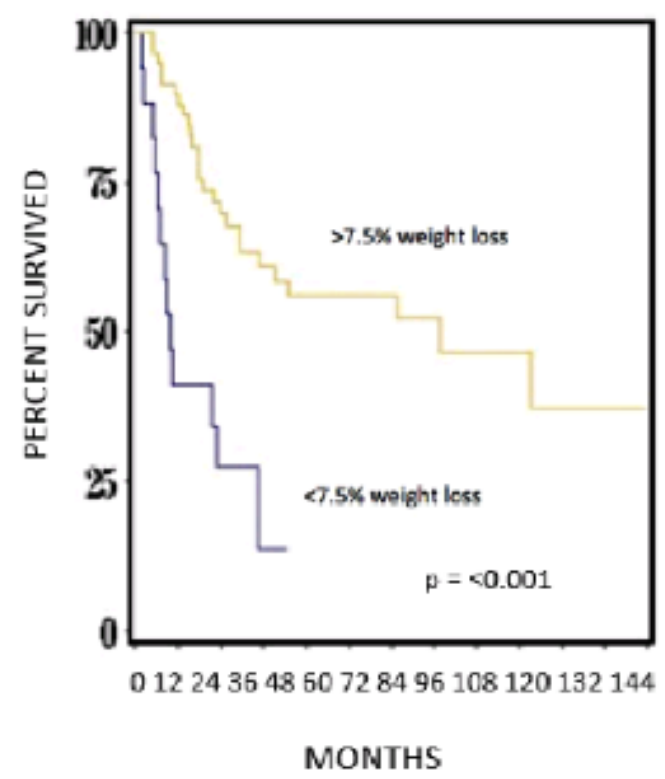

Figure I Kaplan-Meyer Curves for Disease Free and Overall Survival for Severe Treatment Weight Loss (SEVERE) and Non-Severe Treatment Weight Loss (NON-SEVERE) groups.

\section{Discussion}

The results of this study indicate that, independent of pretreatment nutritional status and weight loss, severe weight loss and acute malnutrition during CTRT for Stage III and IV SCCHN not only does not deleteriously affect CTRT toxicity, tumor response, or the need for radical surgery, but, conversely, is associated with increased overall survival and disease-free survival. Toxicity to CTRT did not vary significantly between SEVERE and NON-SEVERE. Primary tumor response to CTRT was numerically superior in SEVERE compared with NON-SEVERE, with CCR 's and HCR's of 71\% vs $45 \%$. Cancer recurrence did not vary among SEVERE versus NONSEVERE. All recurrences were local/regional. These outcomes were not related to unequal distribution of primary tumor sites, as anatomic location differed only in higher hypopharynx/tongue for SEVERE patients, a site that is associated with worsened outcomes. Both overall survival and disease-free survival were increased three to four-fold for SEVERE compared with NON-SEVERE with median survival time for disease free and overall at 83.8 months vs 12.5 months, respectively $(\mathrm{p}<0.002)$. Our review of the literature indicates that this increased survival of SCCHN patients, independent of pretreatment weight/weight loss and nutritional status, who became severely malnourished during CTRT, without concomitant excessive toxicity or need for radical surgery, has not been reported previously, and is a significant finding of this study.

Toxicities such as mucositis, xerostomia, changes in smell/ taste, and dysphagia frequent among patients undergoing combined modality treatment for advanced SCCCHN. ${ }^{10}$ These adverse effects may contribute to increased weight loss. In the present investigation, National Cancer Institute toxicity grades did not vary between SEVERE and NON-SEVERE patient. While similar group toxicities likely result from the controlled CTRT treatment regimen, these results eliminate increased CTRT adverse events as the cause of SEVERE weight loss or of cancer outcomes.

Clinical and histologic tumor responses also did not vary between SEVERE and NON-SEVERE. Pedruzzi et al. ${ }^{11}$ studied a variety of treatment modalities and determined that a radiotherapy dose of $>70$ Gy alone is related to a higher rate of complete response and overall survival compared to lower doses with or without concomitant or neoadjuvant chemotherapy. ${ }^{11}$ The number of patients who required radical surgery for residual disease following CTRT did not differ SEVERE versus NON-SEVERE in the current report. The associated morbidities and potential mortality of radical head and neck surgery, therefore, did not adversely affect survival outcomes.

Local tumor recurrence is not uncommon in patients with advanced stage head and neck cancer. SCCHN recurred in 13/52 (25\%) patients in the present study, $11 / 13(85 \%)$ of which were local. Metachronous cancers developed in 1/11 (9\%) NON-SEVERE and 12/41 (29\%) SEVERE $(\mathrm{p}=0.14)$. Recurrent SCCHN relates to stage, treatment regimen, and/or primary tumor location. ${ }^{12}$ Among 2,067 SCCHN patients Carvalho et al. identified significant variation between tumor stage (I-IV) and treatment modality used. ${ }^{12}$ The present investigation involved only patients with advanced Stage III/IV SCCHN, who, with narrow variation in compliance, underwent an essentially identical and standardized treatment protocol. That recurrence did not differ between SEVERE and NON-SEVERE, in spite of extreme weight loss variation during CTRT, may relate to consistent cancer management.

Patients who develop malnutrition during treatment have been observed to have a higher mean pre-treatment BMI. ${ }^{10,13}$ In our study population, no association was identified between pre-treatment BMI 
and weight loss during treatment. Instead, primary tumor site, more specifically the tongue/hypopharynx, was found to be a significant predictor of severe weight loss in our population ( $p$ 0.017). As documented in previous studies, patients with supraglottic primary tumors of the tongue/hypopharynx represent a cohort that statistically loses more weight during treatment. ${ }^{14,15}$

Ghadjar et al. ${ }^{3}$ reported that pre-treatment weight loss of $>10 \%$ is strongly associated with decreased survival outcomes $(p<0.001){ }^{3}$ Interestingly, they observed no association of weight loss during treatment with survival outcomes in. In another study of 1,168 SCCHN patients undergoing radiation therapy, a critical weight loss of $>5 \%$ during treatment was associated with lower rates of disease-free survival. ${ }^{6}$ Cho et al. ${ }^{15}$ found that disease free survival was significantly higher in patients who did not lose $>10 \%$ of their body weight during and within one year of treatment while Pai et $\mathrm{al}^{2}$ did not find any relationship between weight loss during radiation therapy and overall survival within the 1,562 patient population studied. ${ }^{14,2}$ It is clear from our literature search that the data reported about weight loss during treatment and survival outcomes is inconsistent. The population of patients in the studies reviewed did not have equivalent diagnoses or treatment protocols as did the patients in our study. Our patient population was limited to stage III and IV clinically operable SCCHN and underwent a standardized treatment regimen of concomitant CTRT as described above. ${ }^{6}$

There are several limitations to this investigation. It was a retrospective analysis of prospectively collected data, which carries the usual confounders. Some of the data was self-reported by patients such as pre-treatment weight loss. The power of this study is limited by the population size. Some patients were lost to follow up.

\section{Conclusion}

In spite of greater tongue/hypopharynx primaries and independent of pre-treatment weight loss, CTRT toxicity, post-chemo-radiotherapy operations and tumor recurrence did not vary among SEVERE versus NON-SEVERE. Complete clinical response and histologic responses were excellent in the SEVERE cohort. Most importantly overall and disease-free survivals were increased significantly in SEVERE patients. Our data clearly demonstrated a statistically and clinically significant disease-free and overall survival advantage related to $>7.5 \%$ body weight lost during, but not before CTRT. The pathophysiological mechanisms underlying these new and clinically important findings are not clear from the data. Understanding the implications of these results for the nutritional management of Stage III and IV SCCHN patients who undergo CTRT as the first line cancer treatment will require further investigation.

\section{Acknowledgments}

The Southern New Jersey Head and Neck Cancer Treatment Network of participating physicians' list is provided in the Acknowledgement section.

\section{Funding}

None.

\section{Conflicts of interest}

None.

\section{References}

1. White JV, Guenter P, Jensen G, et al. Consensus Statement: Academy of Nutrition and Dietetics and American Society for Parenteral and Enteral Nutrition: Characteristics Recommended for the Identification and Documentation of Adult Malnutrition (Undernutrition). J Parenter Enter Nutr. 2012;36(3):275-283.

2. Pai PC, Chuang CC, Tseng CK, et al. Impact of pretreatment body mass index on patients with head-and-neck cancer treated with radiation. Int $J$ Radiat Oncol Biol Phys. 2012;83:e93-e100.

3. Ghadjar P, Hayoz S, Zimmermann F, et al. Impact of weight loss on survival after chemoradiation for locally advanced head and neck cancer: Secondary results of a randomized phase III trial. Radiation Oncology. 2015;10:21.

4. Sheck C, Tyrrell J, Davis M, et al. Survival is increased in patients developing severe weight loss during concomitant chemotherapy and radiation therapy for advanced operable stage III and IV squamous cell carcinoma of the head and neck. Otolaryngol Open J. 2017;SE(6):S17-S23.

5. Languis JAE, Bakker S, Rietveld DHF, et al. Critical weight loss is a major prognostic indicator for disease-specific survival in patients with head and neck cancer receiving radiotherapy. British Journal of Cancer. 2013;109:1093-1099.

6. Davis M, Tyrrell J, Slotman G, et al. Preoperative simultaneous fractionated cisplatin and radiation therapy in the treatment of advanced operable stage III and IV squamous cell carcinoma of the head and neck. Am J Surg. 2015;209(3):575-579.

7. Edge SB, Byrd DR, Compton CC, et al. AJCC Cancer Staging Manual. 7th ed. New York: Springer. 2010

8. Common Terminology Criteria for Adverse Events (CTCAE) Version 4.0 Published: May 28, 2009 (v4.03: June 14, 2010) U.S. DEPARTMENT OF HEALTH AND HUMAN SERVICES National Institutes of Health National Cancer Institute.

9. SAS Institute Inc. SAS/STAT ${ }^{\circledR} 9.22$ User's Guide. Cary, NC: SAS Institute Inc. 2010.

10. Zhao JZ, Zheng H, Li LY, et al. Predictors for weight loss in head and neck cancer patients undergoing radiotherapy: a systematic review. Cancer Nurs. 2015;38(6):E37-E45.

11. Pedruzzi PAG, Kowalski LP, Nishimoto IN, et al. Analysis of Prognostic Factors in Patients With Oropharyngeal Squamous Cell Carcinoma Treated With Radiotherapy Alone or in Combination With Systemic Chemotherapy. Arch Otolaryngol Head Neck Surg. 2008;134(11):11961204.

12. Ewa Silander, Jan Nyman, Eva Hammerlid. An exploration of factors predicting malnutrition in patients with advanced head and neck cancer. The Laryngoscope. 2013;123(10):2428-2434.

13. Ottosson S, Zackrisson B, Kjellén E, et al. Weight loss in patients with head and neck cancer during and after conventional and accelerated radiotherapy. Acta Oncologica. 2013;52(4):711-718.

14. Lønbro, S, Petersen, GB, Andersen JR, et al.. Prediction of critical weight loss during radiation treatment in head and neck cancer patients is dependent on BMI. Support Care Cancer. 2016;24(5):2101-2109.

15. Cho YW, Roh JL, Kim SB, et al. Prediction of posttreatment significant body weight loss and its correlation with disease-free survival in patients with oral squamous cell carcinomas. Nutr Cancer. 2013;65(3):417-423.

Citation: Tamburrini D, Sheck CG, Shaikh S, et al. Severe weight loss during, but not before, simultaneous chemotherapy and radiation therapy for stage III and IV squamous cell carcinoma of the head and neck (SCCHN) is associated with increased survival.J Otolaryngol ENT Res. 202I;I3(3):54-58. DOI: 10.15406/joentr.202I.13.0049I 